\title{
Lunar occultations of stars with exoplanet candidates
}

\author{
A. Richichi ${ }^{\star}$ \\ European Southern Observatory, Karl-Schwarzschildstr. 2, 85748 Garching bei München, Germany \\ Received 3 September 2002 / Accepted 29 October 2002

\begin{abstract}
We investigate the potential of lunar occultations for the detection of very faint companions, and in particular for the possibility of detecting extrasolar planets. We review the basic concepts of the method, and discuss the quantities which are relevant for this particular application. The near-infrared range, and in particular the $K$ and $L$ bands (2.2 and $3.6 \mu \mathrm{m})$, offer the best dynamic range. We present results of detailed simulations based on previous experience with lunar occultation events, and taking into account a realistic noise model which includes the effects of detector, lunar background, scintillation. We conclude that lunar occultations at a large telescope ( $8-10 \mathrm{~m}$ class) can detect companions $\approx 5$ to $\approx 11$ mag fainter than the primary, at distances of order $0{ }^{\prime} 01$. The detection is highly asymmetric, with scintillation and photon noise from the central star being the limiting factor in one half-plane of the sky. Although this performance is not strictly sufficient for the direct detection of hot Jupiters, the method can nevertheless provide a valuable check with a relatively simple effort. We provide a list of lunar occultation events of candidate exoplanets visibile from the major observatories in the period 2003-2008. The method would be particularly attractive with large telescopes in the 30 to $100 \mathrm{~m}$ class.
\end{abstract}

Key words. methods: observational - occultations - stars: planetary systems - stars: individual: TMR 1C stars: individual: HD 222582 - stars: pulsars: individual: PSR B1620-26

\section{Introduction}

The search and study of extra-solar planets (or exoplanets for brevity) is one of the fastest growing fields in astronomy. The interpretation of the period variations of the pulsar PSR1257+12 (Wolszczan \& Frail 1992) in terms of perturbations by orbiting planets was the first convincing evidence of the existence of other planetary systems outside our own. Subsequently, the report of a possible exoplanet around $51 \mathrm{Peg}$ (Mayor \& Queloz 1995) paved the way for a wealth of results from a number of sensitive radial velocity surveys. Currently, the number of candidate exoplanets is close to one hundred, and includes detections also by other techniques such as photometric variations due to the transit over the stellar disc, and fine perturbations in the lightcurves of microlensing events. At present, there is a general consensus that many, if not all, of these candidates are indeed exoplanets. Because of selection effects intrinsic in the detection methods, these are all relatively massive objects in close orbit around the parent star, hence the term hot Jupiter that is often used in the literature. In this paper we will refer implicitly to this particular class of exoplanets.

Considerable resources are being invested to expand our knowledge on these objects. The main obstacle is that their direct detection is severely hampered by the huge difference in intensity with respect to the nearby parent stars. Specialized techniques are being developed to limit this disadvantage, including nulling interferometry from the ground and from space,

\footnotetext{
^ e-mail: arichich@eso.org
}

as well as other methods similarly based on destructive interference, including properly modified adaptive optics systems and dark-speckle interferometry. Other techniques, such as radial velocities surveys, photometric transits and microlensing events, are also being improved and expanded. Reviews on this subject were provided for example by Perryman (2000) and Schneider (1999).

In this paper, we concentrate our attention on the possibility of lunar occultations of the parent stars, with the aim of determining the best wavelength range, the technical requirements and the limiting brightness ratio for the detection of faint companions. We provide a list of events observable in the near future from major ground observatories.

\section{The method}

In the following we give a brief description of the lunar occultation (LO) method and of its potential for ground-based observations. Note that the observation of LO from space and their application to exoplanet detections has been presented elsewhere (Richichi 2000). A LO phenomenon can be schematically described as in Fig. 1. As the Moon moves against the background of fixed stars, the target star is subject to a disappearance and a reappearance, marked $S_{\mathrm{D}}$ and $S_{\mathrm{R}}$ in the figure. These usually occur one on the dark and one on the bright limb, depending on the lunar phase. For most practical purposes, only dark-limb events are useful. Fortunate cases in which both $S_{\mathrm{D}}$ and $S_{\mathrm{R}}$ occur on the dark limb are possible, but rare. The Moon moves with a speed $V_{M}$ which is typically $\approx 0.75 \mathrm{~m} / \mathrm{ms}$, or $\approx 0$ '. $4 / \mathrm{s}$. 


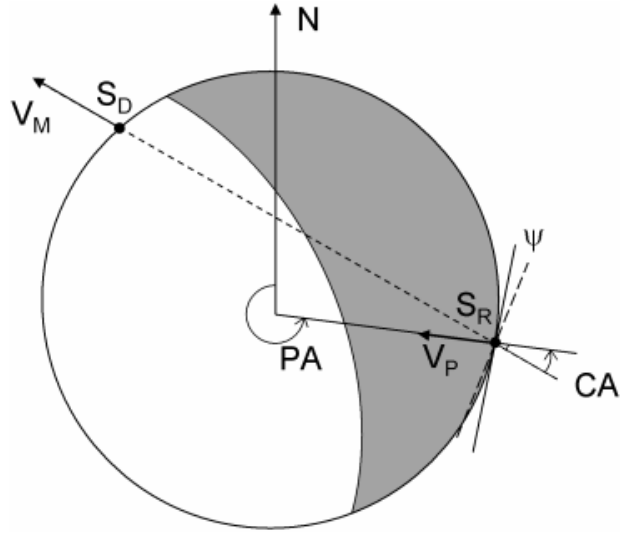

Fig. 1. Schematic representation of a lunar occultation. The quantities are described in the text.

As a result, the lunar limb scans the source with a speed $V_{\mathrm{P}}$ whose module depends on the contact angle CA. The orientation of the scan is determined by the position angle of the event, PA. Both CA and PA are subject to a (small) correction in case a local limb slope $\psi$ is present. Experience on a large number of LO events shows that in general $|\psi| \lesssim 10^{\circ}$.

The lunar limb can be considered for all practical purposes as a straight diffracting edge. The occultation then produces a series of diffraction fringes. As the Moon scans the source, a characteristic pattern of varying intensity is observed on the ground. A few typical examples are provided in Fig. 2. Without entering a discussion of the many details that characterize such lightcurves (see for example Richichi et al. 1996 and references therein), we note here a few factors which are relevant for our purposes.

The bandwidth of the filter and the central wavelength affect the contrast and the frequency of the fringes, as shown in curves a) through c) of Fig. 2. The presence of one or more faint companions results in a superposition of diffraction patterns with corresponding intensities and separated in time, as shown in curve d) of Fig. 2. Note that the angular resolution achieved by LO is not directly linked to the diameter of the telescope used. An important role is played by the sampling of the lightcurve (obviously related to $V_{\mathrm{P}}$ ), and the signal-to-noise ratio (SNR). Angular resolutions at the level of 1 milliarcsec (mas) are routinely achieved also with relatively small telescopes.

Since the fringe pattern is generated at the lunar limb in vacuum, in principle the detection of nearby companions by LO is not affected by the residual illumination in the pointspread function of the central star, which represents a strong limitation for conventional imaging. If observed from space, LO could in fact be considered as a sort of coronographic technique (Richichi 2000). From the ground, the situation is considerably worse. Apart from detector noise, the two main contributions to the SNR are the photon noise and the scintillation noise. This latter can be effectively limited in several ways: by using large apertures on the sky, telescopes of large diameters, and by observing at long wavelengths. Richichi et al. (1992) have also implemented a method to model scintillation noise, and used it to reduce considerably its influence in the analysis

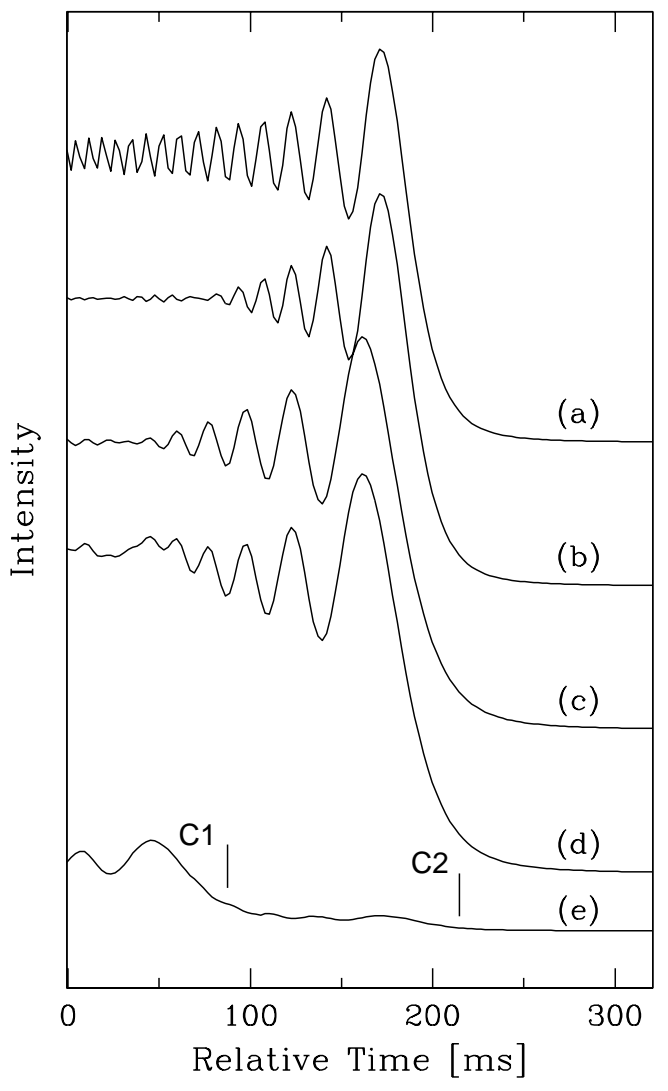

Fig. 2. Illustration of typical LO lightcurves. In all cases, point-like sources are considered, and effects of smearing due to telescope aperture and integration time are neglected. The curves are noise-free. The intensities are normalized and offset by arbitrary amounts. We have assumed a sampling time of $2.4 \mathrm{~ms}$ and $V_{\mathrm{P}}=0.43 / \mathrm{s}$, both typical values. a) single source, monochromatic filter at $2.2 \mu \mathrm{m}$. b) same, with wideband $K$ filter. c) same, with wide-band L filter. d) same as c), with two companions added: $\mathrm{C} 1$ and $\mathrm{C} 2$ with separations -0 ' $^{\prime} 050$ and $+0{ }^{\prime} 005$ and brightness ratios to the primary of 1:10 and 1:50 respectively. e) difference d)-c), enlarged by a factor 2 and with the positions of $\mathrm{C} 1$ and $\mathrm{C} 2$ marked.

of LO data. Photon noise is due to both the background and the light from the occulted star. The background is often dominant in ground-based observation, due mainly to scattered moonlight. Since moonlight has a solar spectrum, this background is strongly wavelength dependent $\left(\approx \lambda^{-4}\right)$. At longer wavelengths, the thermal emission from the sky (and the telescope) also becomes significant and, at wavelengths beyond $\approx 5 \mu \mathrm{m}$, dominant. The use of fast read-out array detectors effectively reduces this source of noise: by using only a small number of pixels, the background is collected only from a relatively small field of view on the sky. Optimal reduction is obtained for pixel sizes matched to the seeing.

\section{Limits of detection}

In order to identify the optimal wavelength range of observation, it is necessary to make realistic assumptions on the relative brightness of potential exoplanets relative to their parent stars. Research in this area is sufficiently advanced by now that models can take into account not just the reflected starlight, 
but also effects of intense irradiation on the planet's atmosphere, as well as the atmospheric chemical composition, the phase of illumination, and even the presence of structures (clouds). It will suffice for our purposes to use orderof-magnitude estimates. Following examples such as those presented by Barman et al. (2001), we can assume that a giant exoplanet heated to about $1000 \mathrm{~K}$ at $0.05 \mathrm{AU}$ from a G2 star could have a flux relative to the star of about $10^{-6}$ in the near-IR. The relative flux improves by about half an order of magnitude from $1 \mu \mathrm{m}$ to $5 \mu \mathrm{m}$. The situation would be more favorable at longer wavelengths, where however the performance of the LO method would be significantly inferior (see Sect. 2). In practice, the best dynamic range would be obtained in the $K$ and $L$ bands ( 2.2 and $3.6 \mu \mathrm{m}$ respectively).

The SNR of LO observations with IR arrays was studied by Richichi (1994, Eq. (1)-(3)). In particular, it was found that LO events with a 4 m-class telescope using an array with $1^{\prime \prime}$ pixel size and $30 \mathrm{e}^{-}$read-out noise $(\mathrm{RON})$, with $12 \mathrm{~ms}$ integration time, could be sensitive to sources as faint as $K \approx 15$ under favorable conditions of moonlight background.

We have refined the model of Richichi (1994), with the inclusion of additional parameters such as scintillation. The number of detector counts from a source of magnitude $K$ and from one square arcsecond of background can be expressed respectively as

$I_{\star} \simeq 10^{(6.84-0.4 K)} \times S$

$B \simeq 10^{(4.59-0.4 \Sigma)} \times S$

where the term $S$ is defined as

$S=\alpha\left(\frac{\epsilon}{25 \%}\right)\left(\frac{\Delta \lambda}{0.4 \mu \mathrm{m}}\right)\left(\frac{\tau}{1 \mathrm{~ms}}\right)\left(\frac{D}{4 \mathrm{~m}}\right)^{2}$

with the obvious factors. The overall transmission and detection efficiency of telescope and instrument is denoted by $\epsilon$, and $\alpha=1 /$ ADU. Following Richichi (1994), we use here the quantity $\Sigma$, which is the $K$ magnitude of the background in a $15^{\prime \prime}$ circular aperture. Typically $2 \leq \Sigma \leq 5$, with values as low as $\Sigma \sim 7$ observed under favorable conditions. The number of noise counts can be expressed as

$N=\sqrt{\alpha^{2} \mathrm{RON}^{2}+\left(\kappa I_{\star}\right)^{2}+\left(I_{\star}+\left(\frac{\sigma}{15^{\prime \prime}}\right)^{2} B\right)}$

where the first term represents the contribution of the RON, the second term is due to the scintillation (expressed as a fraction $\kappa$ of the star light), and the last term to photon shot noise. It is assumed that the background and RON are collected only from as many pixels as required to cover the seeing disk $\sigma$. Note that $N$ is a function of time, since the last two terms in Eq. (4) depend on the stellar intensity which is varying due to the occultation phenomenon. We neglect here the contribution to the noise from the companion. The SNR is then given by $I_{\star} / N$.

We have also revised most of the technical parameters, given the technological improvements achieved at present and foreseeable in the near future. Infrared arrays have been developed for adaptive optics applications with very low RON. We make the assumption that such detectors will be available with
Table 1. Parameters of the simulations.

\begin{tabular}{lrr}
\hline \hline Parameter & Case 1 & Case 2 \\
\hline Filter $\lambda_{0} / \Delta \lambda(\mu \mathrm{m})$ & $2.2 / 0.4$ & $2.2 / 0.4$ \\
Parent star brightness $(K \mathrm{mag})$ & 4.0 & 7.0 \\
Seeing & $0{ }^{\prime} 8$ & $0 .^{\prime} 4$ \\
Pixel size & $0{ }^{\prime} 5$ & $0{ }^{\prime} .3$ \\
RON $\left(\mathrm{e}^{-}\right)$ & 3 & 1 \\
Telescope diameter $(\mathrm{m})$ & 8 & 10 \\
Integration time $(\mathrm{ms})$ & 5 & 20 \\
Background in $15^{\prime \prime}(\Sigma)$ & 5.0 & 7.0 \\
Scintillation $(\kappa)$ & $1 \%$ & $0.5 \%$ \\
Telescope+Optics Transm. & $16 \%$ & $22 \%$ \\
Detector Q.E. & $60 \%$ & $70 \%$ \\
SNR of companion & 1.0 & 1.0 \\
\hline
\end{tabular}

$1-3 \mathrm{e}^{-}$RON. Another significant contribution will be made by the size of the telescope, and its ability to obtain sharp images. Several telescopes of the $8-10$ m class will be in the path of LO events of candidate exoplanets in the near future (see Sect. 4). In parallel with the telescope size, modern technology and the choice of good observing sites are significantly improving the average image quality. We can safely assume that this latter can be at the level of 0.5 for large modern telescopes.

We have considered two cases, which are representative of an average and a very good situation respectively. The parameters of these two test cases are listed in Table 1, and the results are shown in Fig. 3. We note that the two brightest stars with a candidate exoplanet have $V<4$, and that the magnitude distribution peaks around $V \approx 7.5$. Considering that on average the stars have spectral types close to solar, the $K$ magnitudes will be 1-1.5 mag brighter than the $V$ ones. As a result, the two test cases of Table 1 are well representative of the sample. We also note that while an extensive database exists on the performance of the LO technique in the $K$ band for various telescopes, detectors, and lunar phases, unfortunately no comparable detailed database exists for $L$-band observations. Therefore, it is difficult to provide reliable simulations in this latter band.

The detection threshold is expressed as the magnitude difference of the companion with respect to the primary. The threshold is a function of time, due to the varying stellar signal and to scintillation. With large telescopes scintillation is expected to be quite small, but it is nevertheless the limiting factor in the regime in which the stellar light is present. As the light of the central source begins to fade, scintillation noise diminishes progressively, and eventually the limiting factor is set by the combination of RON, pixelsize, and brightness of the background. This latter is dominated by the scattered moonlight, and two different values have been assumed in the simulations.

Figure 3 shows that the process of detection is not symmetric around the central source, and the direction of lunar motion and the PA of the event determine two half-planes, in one of which the detection threshold can be up to 5-6 mag more favorable than in the other. The detection of hot exoplanets, within a fraction of $\mathrm{AU}$ from the parent star, implies separations of few tens of milliarcseconds. This is approximately compatible with the range over which LO events are highly sensitive, but 
Table 2. Occultation events and their characteristics.

\begin{tabular}{clcrrrr}
\hline \hline $\begin{array}{c}(1) \\
\text { Source }\end{array}$ & Obs. & UT Date & $\begin{array}{c}\text { Phase } \\
\text { Un }\end{array}$ & \multicolumn{1}{c}{$\begin{array}{c}\text { P } \\
\text { PA }\end{array}$} & \multicolumn{1}{c}{ Rate } \\
\hline TMR 1C & Par & $06-09-0410: 28$ & $-51 \%$ & 1.59 & $207^{\circ}$ & $-0.212^{\prime \prime} / \mathrm{s}$ \\
HD 222582 & Can & $24-10-0422: 47$ & $90 \%$ & 1.22 & $45^{\circ}$ & $0.383^{\prime \prime} / \mathrm{s}$ \\
HD 222582 & Pal & $21-11-0404: 50$ & $71 \%$ & 1.39 & $70^{\circ}$ & $0.371^{\prime \prime} / \mathrm{s}$ \\
HD 222582 & Gra & $21-11-0405: 05$ & $71 \%$ & 1.52 & $88^{\circ}$ & $0.324^{\prime \prime} / \mathrm{s}$ \\
TMR 1C & Can & $20-01-0522: 15$ & $83 \%$ & 1.01 & $17^{\circ}$ & $0.169^{\prime \prime} / \mathrm{s}$ \\
TMR 1C & Pal & $17-02-0505: 32$ & $62 \%$ & 1.29 & $66^{\circ}$ & $0.337^{\prime \prime} / \mathrm{s}$ \\
TMR 1C & Gra & $17-02-0505: 44$ & $62 \%$ & 1.47 & $62^{\circ}$ & $0.347^{\prime \prime} / \mathrm{s}$ \\
TMR 1C & Sao & $31-07-0500: 32$ & $-21 \%$ & 2.00 & $292^{\circ}$ & $-0.357^{\prime \prime} / \mathrm{s}$ \\
PSR B1620-26 & Par & $17-04-0606: 02$ & $-87 \%$ & 1.06 & $231^{\circ}$ & $-0.161^{\prime \prime} / \mathrm{s}$ \\
PSR B1620-26 & Par & $08-07-0607: 14$ & $90 \%$ & 2.92 & $164^{\circ}$ & $0.142^{\prime \prime} / \mathrm{s}$ \\
PSR B1620-26 & Par & $31-08-0623: 39$ & $51 \%$ & 1.05 & $30^{\circ}$ & $0.095^{\prime \prime} / \mathrm{s}$ \\
PSR B1620-26 & Par & $15-01-0709: 39$ & $-15 \%$ & 1.59 & $259^{\circ}$ & $-0.364^{\prime \prime} / \mathrm{s}$ \\
PSR B1620-26 & Par & $27-03-0807: 27$ & $-73 \%$ & 1.05 & $316^{\circ}$ & $-0.275^{\prime \prime} / \mathrm{s}$ \\
PSR B1620-26 & Par & $07-09-0801: 20$ & $45 \%$ & 1.41 & $94^{\circ}$ & $0.354^{\prime \prime} / \mathrm{s}$ \\
\hline
\end{tabular}

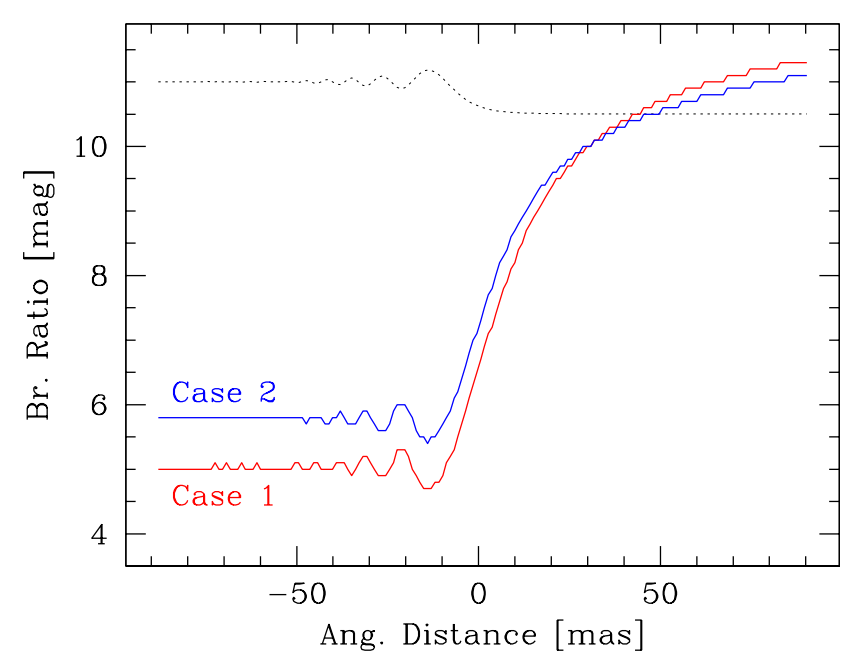

Fig. 3. Detection threshold for a faint companion, for the two different cases listed in Table 1. The horizontal axis is marked in units of the angular distance of the companion from the parent star. The dotted line at the top shows for reference the normalized light curve of the occultation of the parent star. The total span is about $0.4 \mathrm{~s}$. The apparent cross-over of the two curves in the low-threshold regime is due to the increased relative effect of detector noise in Case 2 (i.e., fainter star).

of course the exact computation depends on the distance to the star and on the rate of motion of the lunar limb.

It appears that this method does not have, at least from the ground, the potential for a direct detection of true exoplanets. However, it has sufficient capability to discern between different hypothesis in those cases in which a candidate exoplanet is not the only explanation for a given observational feature. Alternatively, a negative detection can indeed reinforce the reality of the exoplanet hypothesis. Furthermore, the method and the data reduction are very simple and relatively economical in terms of time and resources. The main drawback consists in the fact that, as always with lunar occultations, the source, location and time of the event are fixed and determined by the orbit of the Moon.
It is interesting to compare these results with the capabilities of an ideal space experiment such as that described by Richichi (2000). In the absence of scintillation and background noise, space observations can extend dramatically the range of angles over which a companion can be searched for. At angles as wide as $1^{\prime \prime}$, the detection threshold can then exceed $10^{-6}-10^{-6}$, depending on wavelengths and integration time. On the scale of the small angles over which the ground-based observations would be effective, the difference with space-based observations is only about 1 order of magnitude. Another interesting feature that could be made available by space observations would be that of much longer integration times, if the orbit of the telescope is chosen to decrease substantially the rate of occultation.

When searching for companions which are intrinsically faint, one should consider the possibility of spurious detections of background objects. For Case 2 of Fig. 3, the faintest companions that could be detected would have $K \approx 18$. Assuming an average spectral type $K V$ of the background stars, we are interested in determining the probability of confusion at the level of $V<21$. Using the mean star density at all galactic latitudes (Zombeck 1990) since the parent stars are in the solar neighbourhood, we obtain less than 0.004 stars per square arcsecond.

\section{Calendar of favorable events and discussion}

We have used a compilation of 103 stars listed in Schneider's Catalog of exoplanets candidates as of July 2002, to compute predictions of LO events. We have restricted the search to events observable from observatories with large telescopes. These included: the SAO 6 m telescope in Russia; the La Palma site in the Canary Islands, which in addition to the WHT and TNG telescope in the $4 \mathrm{~m}$ class, will host the $10.4 \mathrm{~m}$ Grantecan; the four $8.2 \mathrm{~m}$ telescopes located on Cerro Paranal in Chile (with some approximation, events computed for Paranal could be observed with the Gemini South telescope as well); in the United States, the sites of M. Graham (LBT) and M. Palomar (Hale). In Table 2, the following codes have been used: Sao, Can, Par, Gra, Pal, in the order given for the sites above. 
Unfortunately no favorable events were found to be observable from Mauna Kea.

Further constraints applied in the predictions were for darklimb events occurring at airmasses $z \leq 3$, with the Sun at least $5^{\circ}$ below the horizon. Also excluded were events with a lunar phase of more than $90 \%$, or taking place on a part of the dark limb very close to the terminator, in order to avoid situations of very high background. The predictions spanned the years from 2003 through 2008, included.

A total of 14 events for 3 sources were found, and are listed in Table 2. In the table, after the name of the source and the observatory code mentioned above, the UT date and time is provided, rounded to the nearest minute. More accurate predictions can be provided by the author on request. Column 4 lists the fraction of the sunlit lunar disc, with positive and negative numbers indicating disappearances and reappearances respectively. Column 5 is the airmass, Col. 6 the position angle of the event, and Col. 7 the rate at which the limb will scan the source, expressed in "/s.

One may note that, although our predictions have been based on the list of currently known exoplanet candidates, the method could be profitably applied also to all stars which are currently the object of radial velocity surveys, thereby significantly increasing the number of LO events accessible to large telescopes. Additionally, one could consider the application of this technique also to other types of faint companions, such as brown dwarfs. Since catalogs of objects being searched for these types of companions are in constant evolution, it is impractical to include in this paper a complete list of additional predictions. The author is available to compute predictions on request.

\section{Conclusions}

We have discussed the possibility of detection of very faint companions by means of lunar occultation (LO) observations, and concluded that the $K$ and $L$ bands offer the highest dynamical range. We have computed the detection threshold using a detailed noise model, based on previous experience with LO data, and assuming top conditions in terms of telescope, technical equipment and site. The result is that LO might detect companions $\approx 11 \mathrm{mag}$ fainter than the central star, as close as $\approx 0$. 01 . The probability of spurious detection of background stars is found to be very small. The detection probability is not symmetrical, and in the half-plane of the sky where the central star is not occulted the threshold can be $\approx 5$ mag higher. The limiting factors are scintillation (which defines the minimum threshold) and the combination of RON, pixelsize, integration time and intensity of background (which define the maximum threshold). The performance of LO in this context would not be generally sufficient to detect hot exoplanets directly. However, it could set constraints on their brightness, or even provide alternative explanations in those cases in which a margin of doubt still exists. We have provided a table of predictions for 14 events involving 3 exoplanet candidates visibile from the largest observatory in the period 2003-2008.

The size of the telescope, as well as continuous progress in the technical specifications of the detectors, are critical to turn the results of this method from upper limits to direct detections. In this sense, the development of ground-based telescopes with diameters of several tens of meters would be a decisive factor. Simulations with a $100 \mathrm{~m}$ telescope show an increase of at least two magnitudes in the detection threshold for a faint companion with respect to the case of an $8 \mathrm{~m}$ telescope. This, in connection with the availability of better detectors, would provide the capability to detect faint companions with a flux of $10^{-6}$ of the primary.

Acknowledgements. This research has made use of the Simbad database, operated at the Centre de Données Astronomiques de Strasbourg (CDS), and of NASA's Astrophysics Data System Bibliographic Services (ADS). The list of stars with exoplanet candidates was taken from J. Schneider's catalog available at http://www.obspm.fr/encycl/catalog.html.

\section{References}

Barman, T. S., Hauschildt, P. H., \& Allard, F. 2001, ApJ, 556, 885

Mayor, M., \& Queloz, D. 1995, Nature, 378, 355

Perryman, M. 2000, Rep. Progress Phys., 63, 1209

Richichi, A., Di Giacomo, A., Lisi, F., \& Calamai, G. 1992, A\&A, 265,535

Richichi, A. 1994, IAU Symp. 158, ed. W. J. Tango, \& J. G. Robertson, 71

Richichi, A., Baffa, C., Calamai, G., \& Lisi, F. 1996, AJ, 112, 2786

Richichi, A. 2000, ESA SP-451, p. 205

Schneider, J. 1999, C.R. Acad. Sci. Paris, 327, Serie II b, 621

Wolszczan, A., \& Frail, D. A. 1992, Nature, 355, 145

Zombeck, M. V. 1990, Handbook of space astronomy and astrophysics, 2nd ed. (Cambridge University Press) 